\title{
Meta-Analysis the Effect of Constraint-Induced Movement Therapy on Hand Function and Occupational Performance in Children with Cerebral Palsy
}

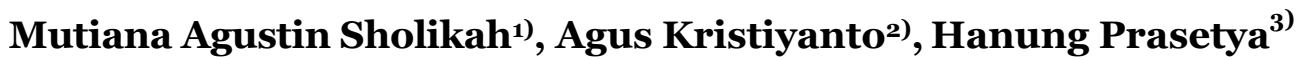 \\ ${ }^{1)}$ Masters Program in Public Health, Universitas Sebelas Maret \\ 2)Faculty of Sport Education, Universitas Sebelas Maret \\ 3)Health Polytechnics, Ministry of Health Surakarta
}

\section{ABSTRACT}

Background: Children with cerebral palsy generally experience hand function which then affect the child's occupational performance. Hand function is the ability to use the hand in doing activities. To be able to perform occupational performance, good hand function are needed. To achieve this, interventions are needed, one of which is constraint-induced movement therapy (CIMT) which is hypothesized to be able to improve hand function and occupational performance. The purpose of this study was to determine the effect of CIMT on the hands function and the occupation performance of children with cerebral palsy.

Subjects and Method: This study used a systematic review design and meta-analysis using the PRISMA flow chart guidelines. The process of searching for articles was carried out between 2005-2020 using databases from PubMed, Science Direct, AJOT, Springer Link, and Google Scholar. Based on the database, there were 20 articles that met the inclusion criteria. This study involved 669 subjects of hand function and 440 subjects of occupational performance. The analysis was performed using the RevMan 5.3 software.
Results: A total of 20 articles conducted a meta-analysis review. A total of 18 articles conducted a meta-analysis showing that giving CIMT to children with cerebral palsy was significantly able to improve hands function of children with cerebral palsy (SMD $=0.40 ; 95 \%$ CI 0.09 to $0.71 ; p=0.01)$. A total of 11 articles conducted a meta-analysis showing that giving CIMT to children with cerebral palsy was able to improve occupational performance abilities but it was not statistically significant (SMD = $0.18 ; 95 \%$ CI -0.17 to $0.52 ; \mathrm{p}=0.32$ ).

Conclusion: CIMT can improve hand function and occupational performance of children with cerebral palsy.

Keywords: constraint-induced movement therapy (CIMT), hand function, occupational performance, cerebral palsy, meta-analysis

\section{Correspondence:}

Mutiana Agustin Sholikah. Masters Program in Public Health. Universitas Sebelas Maret, Jl.Ir. Sutami 36A, Surakarta 57126, Jawa Tengah, Indonesia. Email: mutiana.ash@gmail.com. Mobile: 081215038686.

\section{Cite this as:}

Sholikah MA, Kristiyanto A, Prasetya H (2021). Meta-Analysis the Effect of Constraint-Induced Movement Therapy on Hands Functional Ability and Occupational Performance in Children with Cerebral Palsy. J Epidemiol Public Health. 06(01): 1-11. https://doi.org/10.26911/jepublichealth.2021.06.01.01.

cc (i) (-) Journal of Epidemiology and Public Healthis licensed under a Creative Commons EY NC SA Attribution-NonCommercial-ShareAlike 4.0 International License.

\section{BACKGROUND}

Cerebral palsy is also often associated with stable conditions and non-progressive disorders of the brain of the fetus or developing baby so that they are considered normal children with special needs (Levitt and
Addison, 2019; Miller et al., 2020). The prevalence of cerebral palsy in the world reaches 2 per 1,000 births (Gulati and Sondhi, 2018). So that cerebral palsy is considered the most common cause of 
disability in childhood (Levitt and Addison, 2019).

During development, children with cerebral palsy experience several growth and development disorders such as spasticity, muscle weakness and limitations in activities. More than $50 \%$ of children with cerebral palsy experience problems in the wrist or hand (Ervim in Duruöz, 2019).

Impaired hand function is the most frequent consequence of cerebral palsy in children (Klevberg et al., 2017). Hand function is the ability to use the hand to perform daily activities seen from anatomy, sensory, strength, dexterity and hand coordination (Duruöz, 2019).

When children experience impaired hand function, it is possible that they will experience difficulties in carrying out their daily activities and performance at work and leisure activities (Ervim in Duruöz, 2019). Occupational performance is the ability to perform and complete a series of tasks as a result of an alignment between individuals, the environment and the activity itself (AOTA, 2014).

One of the interventions for children with cerebral palsy is CIMT which is used to treat dysfunctioning limb non-use by limiting healthy limbs and exercising dysfunctional limbs (Christmas et al., 2018). CIMT has 2 key components, namely limb restriction that is less influential and structured and intensive therapy for the arm or hand that has a higher influence (Ilieva and Ilieva, 2020).

In a study by Al-Oraibi and Eliasson (2011), giving CIMT can significantly improve hand function $(\mathrm{p}=0.016)$ using the AHA instrument. In addition, Brandao et al. (2012) stated that giving CIMT is considered to significantly improve children's occupational performance $(p<0.001)$ by using the COPM instrument.
This study is expected to be able to prove the effect of giving CIMT to improve hand function and occupational performance of children with cerebral palsy.

\section{SUBJECTS AND METHOD}

\section{Study Design}

This study uses systematic review and meta-analysis with the PRISMA flow diagram guidelines. Search for articles using databases: PubMed, Science Direct, AJOT, Springer Link, and Google Scholar. Some of the keywords used are: "Constraint Induced Movement Therapy" AND "cerebral palsy", "hand function", "occupational performance", "randomized control trial.

\section{Inclusion Criteria}

The inclusion criteria for this research article were full paper randomized controlled trails (RCT), using CIMT intervention, the subjects were children with unilateral cerebral palsy or hemiplegic aged $<17$ years and outcomes were measured using standardized instruments.

\section{Exclusion Criteria}

The exclusion criteria for this research article were articles that were not in English, published before 2005, comparing the dose of intervention.

\section{Operational Definition of Variables}

Articles included in the study were adapted to the PICO. The article search was carried out by considering the eligibility criteria defined using the PICO model. The study population was children with cerebral palsy, using CIMT intervention and nonCIMT control and the outcomes were an increase in hand function and occupational performance.

Hand function is the ability to use the hands to perform daily activities as measured using standardized instruments.

Occupational performance is a person's ability to carry out productivity activities, 
activity daily living and leisure activities measured using standardized instruments. CIMT is a treatment to treat non-use of sore hands by limiting healthy hands with published article guidelines.

\section{Instruments}

The study used PRISMA flow diagrams and the assessment of the quality of research articles used the Critical Appraisal Checklist for RCT Study tools (CEBM, 2014).

\section{Data Analysis}

The data in this study were analyzed using the RevMan 5.3 application, to calculate the effect size and heterogeneity of the study. The results of data processing are presented in the form of a forest plot and a funnel plot.

\section{RESULTS}

The article review process was carried out using the PRISMA flow chart, which can be seen in Figure 1. The total articles obtained were 20 articles. For the distribution of articles on 4 continents included 7 from Asia, 4 from America, 6 from Europe, and 3 from Australia.

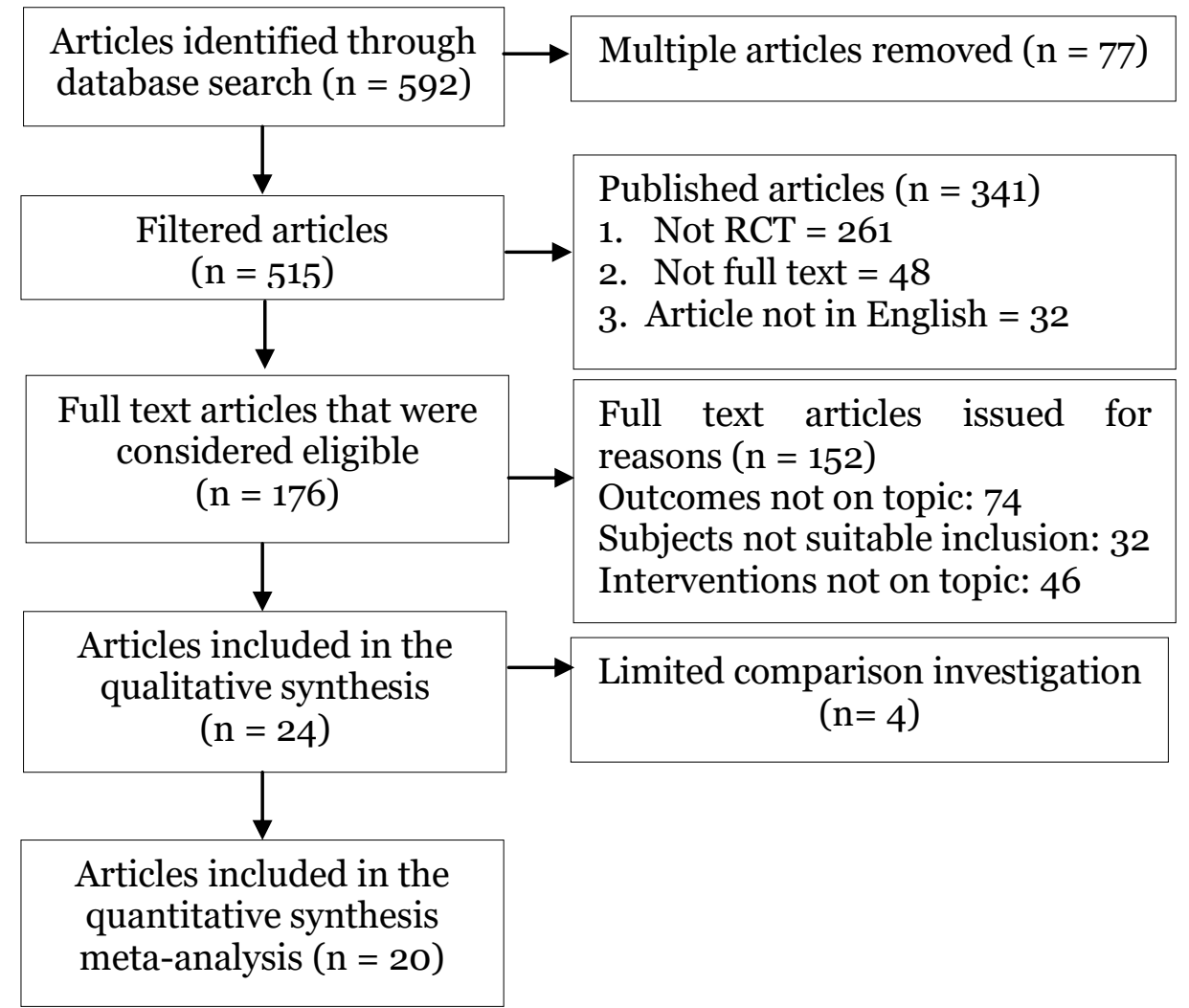

Figure 1. PRISMA flow chart

1. The effect of CIMT on the functional ability of the hands of children with cerebral palsy
Table 1 shows 18 RCT articles about the effect of CIMT on hand function of children with cerebral palsy (CP) that meet the qualitative and quantitative requirements.

Table 1. The effect of CIMT on hand function of children with cerebral palsy 
Sholikah et al./ the Effect of Constraint-Induced Movement Therapy on Hands Functional Ability

\begin{tabular}{|c|c|c|c|c|c|c|c|}
\hline \multirow{2}{*}{$\begin{array}{l}\text { Author, } \\
\text { year }\end{array}$} & \multirow{2}{*}{ Country } & \multirow{2}{*}{$\begin{array}{l}\text { Popula- } \\
\text { tion }\end{array}$} & \multirow{2}{*}{$\begin{array}{c}\text { Instru- } \\
\text { ment }\end{array}$} & \multicolumn{4}{|c|}{ Results } \\
\hline & & & & \multicolumn{2}{|c|}{ Intervention } & \multicolumn{2}{|c|}{ Comparison } \\
\hline $\begin{array}{l}\text { Aarts et al., } \\
2010\end{array}$ & Netherland & $\begin{array}{l}\mathrm{CP} \\
\text { unilateral }\end{array}$ & AHA & $\begin{array}{l}\text { mCIMT } \\
\text {-BiT }\end{array}$ & $\begin{array}{l}\text { Mean : } 60.1 \\
\text { SD : } 15.3\end{array}$ & $\mathrm{UC}$ & $\begin{array}{l}\text { Mean : } 53.1 \\
\text { SD : } 22.2\end{array}$ \\
\hline $\begin{array}{l}\text { Aarts et al., } \\
2011\end{array}$ & Netherland & $\begin{array}{l}\mathrm{CP} \\
\text { unilateral }\end{array}$ & $\begin{array}{l}\text { VOAA- } \\
\text { DDD }\end{array}$ & $\begin{array}{l}\text { mCIMT } \\
\text {-BiT }\end{array}$ & $\begin{array}{l}\text { Mean : } 69.6 \\
\text { SD : } 21.4\end{array}$ & UC & $\begin{array}{l}\text { Mean : } 50.4 \\
\text { SD : } 28.5\end{array}$ \\
\hline $\begin{array}{l}\text { Al Oraibi \& } \\
\text { Eliasson, } \\
2011\end{array}$ & Jordan & $\begin{array}{l}\mathrm{CP} \\
\text { unilateral }\end{array}$ & AHA & CIMT & $\begin{array}{l}\text { Mean : } 48 \\
\text { SD :11.7 }\end{array}$ & NDT/UC & $\begin{array}{l}\text { Mean : } 56.6 \\
\text { SD : } 18.7\end{array}$ \\
\hline $\begin{array}{l}\text { Brandao et } \\
\text { al., } 2010\end{array}$ & Brazil & $\begin{array}{l}\mathrm{CP} \\
\text { hemiplegic }\end{array}$ & JTHF & CIMT & $\begin{array}{l}\text { Mean : } \\
90.48 \\
\text { SD : } 99.83\end{array}$ & $\mathrm{UC}$ & $\begin{array}{l}\text { Mean : } 146.11 \\
\text { SD : } 186.41\end{array}$ \\
\hline $\begin{array}{l}\text { Charles et } \\
\text { al., } 2006\end{array}$ & America & $\begin{array}{l}\mathrm{CP} \\
\text { hemiplegic }\end{array}$ & JTTHF & CIMT & $\begin{array}{l}\text { Mean : } \\
268.6 \\
\text { SD : } 238.0\end{array}$ & UC & $\begin{array}{l}\text { Mean : } 260.3 \\
\text { SD : } 153.0\end{array}$ \\
\hline $\begin{array}{l}\text { Chen et al., } \\
2012\end{array}$ & Taiwan & $\begin{array}{l}\mathrm{CP} \\
\text { unilateral }\end{array}$ & $\begin{array}{l}\text { PMAL- } \\
\text { AOU }\end{array}$ & CIT & $\begin{array}{l}\text { Mean : } 2.5 \\
\text { SD : } 0.3\end{array}$ & TR & $\begin{array}{l}\text { Mean : } 2.3 \\
\text { SD : } 0.3\end{array}$ \\
\hline $\begin{array}{l}\text { Chen et al., } \\
2014\end{array}$ & Taiwan & $\begin{array}{l}\mathrm{CP} \\
\text { unilateral }\end{array}$ & BOTMP-8 & hCIT & $\begin{array}{l}\text { Mean : } 8.87 \\
\text { SD : } 2.26\end{array}$ & TR & $\begin{array}{l}\text { Mean : } 7.86 \\
\text { SD : } 1.64\end{array}$ \\
\hline $\begin{array}{l}\text { Deppe et } \\
\text { al., } 2013\end{array}$ & German & $\begin{array}{l}\mathrm{CP} \\
\text { hemiplegic }\end{array}$ & AHA & CIMT & $\begin{array}{l}\text { Mean : } 58.4 \\
\text { SD : } 9.1\end{array}$ & IBT & $\begin{array}{l}\text { Mean : } 62.3 \\
\text { SD : } 13.5\end{array}$ \\
\hline $\begin{array}{l}\text { Eliasson et } \\
\text { al., } 2005\end{array}$ & Sweden & $\begin{array}{l}\mathrm{CP} \\
\text { hemiplegic }\end{array}$ & AHA & $\mathrm{CI}$ & $\begin{array}{l}\text { Mean : }-1.63 \\
\text { SD : } 2.91\end{array}$ & ST & $\begin{array}{l}\text { Mean : - } 2.06 \\
\text { SD : } 3.11\end{array}$ \\
\hline $\begin{array}{l}\text { Eliasson et } \\
\text { al., } 2011\end{array}$ & Sweden & $\begin{array}{l}\mathrm{CP} \\
\text { unilateral }\end{array}$ & AHA & $\begin{array}{l}\text { Eco- } \\
\text { CIMT }\end{array}$ & $\begin{array}{l}\text { Mean : } 59 \\
\text { SD : } 9\end{array}$ & ST & $\begin{array}{l}\text { Mean : } 56 \\
\text { SD : } 19\end{array}$ \\
\hline $\begin{array}{l}\text { Fedrizzi et } \\
\text { al., } 2013\end{array}$ & Italy & $\begin{array}{l}\mathrm{CP} \\
\text { hemiplegic }\end{array}$ & $\begin{array}{l}\text { Besta } \\
\text { Scale-BU }\end{array}$ & CIMT & $\begin{array}{l}\text { Mean : } 2.74 \\
\text { SD : } 0.84\end{array}$ & ST & $\begin{array}{l}\text { Mean : } 2.97 \\
\text { SD : } 0.68\end{array}$ \\
\hline $\begin{array}{l}\text { Hsin et al., } \\
2012\end{array}$ & Taiwan & $\begin{array}{l}\mathrm{CP} \\
\text { unilateral }\end{array}$ & BOTMP-8 & hCIT & $\begin{array}{l}\text { Mean : } 10.6 \\
\text { SD : } 1.6\end{array}$ & TR & $\begin{array}{l}\text { Mean : } 8.9 \\
\text { SD : } 1.1\end{array}$ \\
\hline $\begin{array}{l}\text { Hwang et } \\
\text { al., } 2020\end{array}$ & South Korea & $\begin{array}{l}\mathrm{CP} \\
\text { unilateral }\end{array}$ & $\begin{array}{l}\text { PMAL- } \\
\text { HW }\end{array}$ & CIMT & $\begin{array}{l}\text { Mean : } 1.83 \\
\text { SD : } 1.12\end{array}$ & CT & $\begin{array}{l}\text { Mean : } 1.85 \\
\text { SD : } 1.03\end{array}$ \\
\hline $\begin{array}{l}\text { Rostami et } \\
\text { al., } 2012\end{array}$ & Iran & $\begin{array}{l}\mathrm{CP} \\
\text { hemiplegic }\end{array}$ & BOTMP-8 & CIMT & $\begin{array}{l}\text { Mean : } 1.35 \\
\text { SD : } 0.37\end{array}$ & Control & $\begin{array}{l}\text { Mean : } 0.28 \\
\text { SD : } 0.8\end{array}$ \\
\hline $\begin{array}{l}\text { Sakzewski } \\
\text { et al., } 2011\end{array}$ & Australia & $\begin{array}{l}\mathrm{CP} \\
\text { unilateral }\end{array}$ & JTTHF & CIMT & $\begin{array}{l}\text { Mean : } 337.5 \\
\text { SD : } 203.8\end{array}$ & BIM & $\begin{array}{l}\text { Mean : } 306.8 \\
\text { SD : } 179.8\end{array}$ \\
\hline $\begin{array}{l}\text { Sakzewski } \\
\text { et al., } 2015\end{array}$ & Australia & $\begin{array}{l}\mathrm{CP} \\
\text { unilateral }\end{array}$ & AHA & CIMT & $\begin{array}{l}\text { Mean : } 62.7 \\
\text { SD : } 13.2\end{array}$ & $\mathrm{SC}$ & $\begin{array}{l}\text { Mean : } 62.2 \\
\text { SD : } 18.4\end{array}$ \\
\hline $\begin{array}{l}\text { Taub et al., } \\
2011\end{array}$ & USA & $\begin{array}{l}\mathrm{CP} \\
\text { hemiplegic }\end{array}$ & PMAL & CIMT & $\begin{array}{l}\text { Mean : } 3 \cdot 5 \\
\text { SD : } 0.6\end{array}$ & UC & $\begin{array}{l}\text { Mean : } 1.4 \\
\text { SD : } 0.5\end{array}$ \\
\hline $\begin{array}{l}\text { Wallen et } \\
\text { al., } 2011\end{array}$ & Australia & $\begin{array}{l}\mathrm{CP} \\
\text { hemiplegic }\end{array}$ & AHA & CIMT & $\begin{array}{l}\text { Mean : } 62.9 \\
\text { SD : } 29.3\end{array}$ & IOT & $\begin{array}{l}\text { Mean : } 52.0 \\
\text { SD : } 28.9\end{array}$ \\
\hline
\end{tabular}

\section{a. Forest plot}

Figure 2 shows that the results of the analysis show that giving CIMT to children with cerebral palsy can increase hand func- tion by 0.4 times than non-CIMT interventions. These results were statistically significant $(\mathrm{SMD}=0.40 ; 95 \% \mathrm{CI} 0.09$ to $0.71 ; \mathrm{p}=$ 0.01). 
Sholikah et al./ the Effect of Constraint-Induced Movement Therapy on Hands Functional Ability

\begin{tabular}{|c|c|c|c|c|c|c|c|c|c|c|c|}
\hline \multirow[b]{2}{*}{ Study or Subgroup } & \multicolumn{3}{|c|}{ Experimental } & \multicolumn{2}{|c|}{ Control } & \multirow[b]{2}{*}{ Total } & \multirow{2}{*}{ Weight } & \multirow{2}{*}{$\begin{array}{l}\text { Std. Mean Difference } \\
\text { IV, Random, } 95 \% \mathrm{Cl}\end{array}$} & \multirow{2}{*}{\multicolumn{2}{|c|}{$\begin{array}{l}\text { Std. Mean Difference } \\
\text { IV, Random, } 95 \% \mathrm{Cl}\end{array}$}} & \\
\hline & Mean & SD & Total & Mean & SD & & & & & & \\
\hline Aarts 2010 & 60.1 & 15.3 & 28 & 53.1 & 22.2 & 22 & $6.6 \%$ & $0.37[-0.19,0.93]$ & & & \\
\hline Aarts 2011 & 69.6 & 21.4 & 28 & 50.4 & 28.5 & 22 & $6.5 \%$ & $0.76[0.18,1.34]$ & & & \\
\hline Al-Oraibi 2011 & 48 & 11.7 & 7 & 56.6 & 18.7 & 7 & $4.2 \%$ & $-0.52[-1.59,0.56]$ & & & \\
\hline Brandao 2010 & 90.48 & 99.83 & 8 & 146.11 & 186.41 & 8 & $4.5 \%$ & $-0.35[-1.34,0.64]$ & & & \\
\hline Charles 2006 & 268.6 & 238 & 11 & 260.3 & 153 & 11 & $5.2 \%$ & $0.04[-0.80,0.88]$ & & & \\
\hline Chen 2012 & 2.5 & 0.3 & 24 & 2.3 & 0.3 & 23 & $6.4 \%$ & $0.66[0.07,1.24]$ & & & \\
\hline Chen 2014 & 8.87 & 2.26 & 23 & 7.86 & 1.64 & 22 & $6.4 \%$ & $0.50[-0.09,1.10]$ & & & \\
\hline Deppe 2013 & 58.4 & 9.1 & 24 & 62.3 & 13.5 & 18 & $6.3 \%$ & $-0.34[-0.96,0.27]$ & & & \\
\hline Eliasson 2005 & -1.63 & 2.91 & 21 & -2.06 & 3.11 & 20 & $6.3 \%$ & $0.14[-0.47,0.75]$ & & & \\
\hline Eliasson 2011 & 59 & 9 & 18 & 46 & 21 & 15 & $5.8 \%$ & $0.81[0.10,1.53]$ & & & \\
\hline Fedrizzi 2013 & 2.74 & 0.84 & 39 & 2.97 & 0.68 & 33 & $7.0 \%$ & $-0.30[-0.76,0.17]$ & & & \\
\hline $\mathrm{H} \sin 2012$ & 10.6 & 1.6 & 11 & 8.9 & 1.1 & 11 & $4.8 \%$ & $1.19[0.27,2.11]$ & & & \\
\hline Hwang 2020 & 1.83 & 1.12 & 12 & 1.85 & 1.03 & 12 & $5.4 \%$ & $-0.02[-0.82,0.78]$ & & & \\
\hline Rostami 2012 & 1.35 & 0.37 & 8 & 0.28 & 0.08 & 8 & $2.2 \%$ & $3.78[1.98,5.58]$ & & & \\
\hline Sakzewski 2011 & 337.5 & 203.8 & 29 & 306.8 & 179.8 & 29 & $6.8 \%$ & $0.16[-0.36,0.67]$ & & & \\
\hline Sakzewski 2015 & 62.7 & 13.2 & 27 & 62.2 & 18.4 & 20 & $6.5 \%$ & $0.03[-0.55,0.61]$ & & & \\
\hline Taub 2011 & 3.5 & 0.6 & 10 & 1.4 & 0.5 & 10 & $2.7 \%$ & $3.64[2.11,5.18]$ & & & \\
\hline Wallen 2011 & 62.9 & 29.3 & 25 & 52 & 28.9 & 25 & $6.6 \%$ & $0.37[-0.19,0.93]$ & & & \\
\hline Total $(95 \% \mathrm{Cl})$ & & & 353 & & & 316 & $100.0 \%$ & $0.40[0.09,0.71]$ & & & \\
\hline \multicolumn{9}{|c|}{$\begin{array}{l}\text { Heterogeneity: } \operatorname{Tau}^{2}=0.29 ; \mathrm{Chi}^{2}=59.23, \mathrm{df}=17(\mathrm{P}<0.00001) ;\left.\right|^{2}=71 \% \\
\text { Test for overall effect: } Z=2.55(\mathrm{P}=0.01)\end{array}$} & -2 & 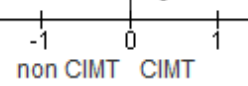 & $\frac{1}{2}$ \\
\hline
\end{tabular}

\section{Figure 2. Forest plot of CIMT's influence on hand function of a child with cerebral palsy}

\section{b. Funnel plot}

In Figure 3, the funnel plot graph looks symmetrical, which shows that there is no publication bias because there are 9 points on the left side, 4 points parallel to the line and 5 points on the right.

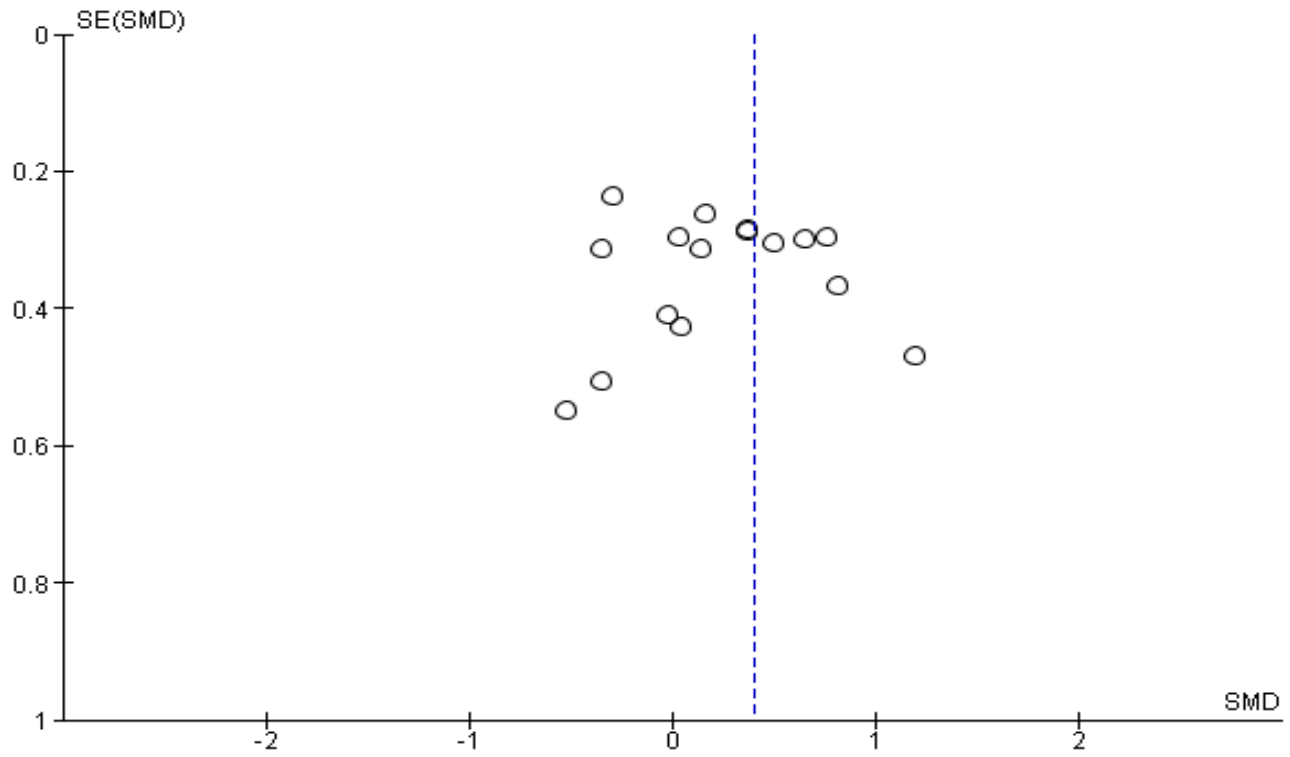

Figure 3. Funnel plot of the effect of CIMT on hand function of a child with cerebral palsy

2. Effect of CIMT on occupational performance of children with cerebral palsy

Table 2 shows 11 randomized control trial (RCT) articles on the effect of CIMT on occupational performance of children with cerebral palsy that meet the qualitative and quantitative requirements. 
Sholikah et al./ the Effect of Constraint-Induced Movement Therapy on Hands Functional Ability

Table 2. Effect of CIMT on occupational performance of children with cerebral palsy

\begin{tabular}{|c|c|c|c|c|c|c|c|}
\hline \multirow{2}{*}{$\begin{array}{c}\text { Author, } \\
\text { Year }\end{array}$} & \multirow{2}{*}{ Country } & \multirow{2}{*}{ Population } & \multirow{2}{*}{$\begin{array}{c}\text { Instru- } \\
\text { ment }\end{array}$} & \multicolumn{4}{|c|}{$\begin{array}{rr}\text { Results } \\
\end{array}$} \\
\hline & & & & Int & rvention & & mparison \\
\hline $\begin{array}{l}\text { Aarts et al., } \\
2010\end{array}$ & Netherland & CP unilateral & COPM-P & $\begin{array}{l}\text { mCIMT- } \\
\text { BiT }\end{array}$ & $\begin{array}{l}\text { Mean : } 6.5 \\
\text { SD : 1.0 }\end{array}$ & $\mathrm{UC}$ & $\begin{array}{l}\text { Mean : } 4.6 \\
\text { SD : } 1.4\end{array}$ \\
\hline $\begin{array}{l}\text { Brandao et } \\
\text { al., } 2010\end{array}$ & Brazil & CP hemiplegic & PEDI-FA & CIMT & $\begin{array}{l}\text { Mean : } 77.36 \\
\text { SD : } 9.32\end{array}$ & $\mathrm{UC}$ & $\begin{array}{l}\text { Mean : } 70.8 \\
\text { SD : } 7.24\end{array}$ \\
\hline $\begin{array}{l}\text { Brandao et } \\
\text { al., } 2012\end{array}$ & Brazil & CP hemiplegic & COPM-P & CIMT & $\begin{array}{l}\text { Mean : } 5.54 \\
\text { SD : } 1.7\end{array}$ & $\begin{array}{l}\text { HABI } \\
T\end{array}$ & $\begin{array}{l}\text { Mean : } 6.58 \\
\text { SD : } 1.19\end{array}$ \\
\hline $\begin{array}{l}\text { Chen et al., } \\
2014\end{array}$ & Taiwan & CP unilateral & WeeFIM & hCIT & $\begin{array}{l}\text { Mean : } 42.61 \\
\text { SD : } 6.24\end{array}$ & TR & $\begin{array}{l}\text { Mean : } 39.73 \\
\text { SD : } 3.66\end{array}$ \\
\hline $\begin{array}{l}\text { Deppe et } \\
\text { al., } 2013\end{array}$ & Jerman & CP hemiplegic & PEDI & CIMT & $\begin{array}{l}\text { Mean : } 62.2 \\
\text { SD : } 8.1\end{array}$ & IBT & $\begin{array}{l}\text { Mean : } 63.2 \\
\text { SD : } 8.3\end{array}$ \\
\hline $\begin{array}{l}\text { Fedrizzi et } \\
\text { al., } 2013\end{array}$ & Italy & CP hemiplegic & $\begin{array}{l}\text { Besta } \\
\text { Scale- } \\
\text { ADL }\end{array}$ & CIMT & $\begin{array}{l}\text { Mean : } 2.4 \\
\text { SD : } 0.95\end{array}$ & ST & $\begin{array}{l}\text { Mean : } 2.38 \\
\text { SD : } 0.83\end{array}$ \\
\hline $\begin{array}{l}\text { Hwang \& } \\
\text { Kwon, } \\
2020\end{array}$ & $\begin{array}{l}\text { South } \\
\text { Korea }\end{array}$ & CP unilateral & PEDI-SC & CIMT & $\begin{array}{l}\text { Mean : } 17.92 \\
\text { SD : } 12.47\end{array}$ & CT & $\begin{array}{l}\text { Mean : } 16.25 \\
\text { SD : } 9.27\end{array}$ \\
\hline $\begin{array}{l}\text { Sakzewski } \\
\text { et al., } 2011\end{array}$ & Australia & $\mathrm{CP}$ unilateral & COPM-P & CIMT & $\begin{array}{l}\text { Mean : } 6.3 \\
\text { SD : } 1.9\end{array}$ & BIM & $\begin{array}{l}\text { Mean : } 6.3 \\
\text { SD : } 1.5\end{array}$ \\
\hline $\begin{array}{l}\text { Sakzewski } \\
\text { et al., } 2015\end{array}$ & Australia & $\mathrm{CP}$ unilateral & COPM-P & CIMT & $\begin{array}{l}\text { Mean : } 6.1 \\
\text { SD : } 1.5\end{array}$ & $\mathrm{SC}$ & $\begin{array}{l}\text { Mean : } 6.9 \\
\text { SD : } 1.0\end{array}$ \\
\hline $\begin{array}{l}\text { Wallen et } \\
\text { al., } 2011\end{array}$ & Australia & CP hemiplegic & COPM-P & CIMT & $\begin{array}{l}\text { Mean : } 6.1 \\
\text { SD : } 2.3\end{array}$ & IOT & $\begin{array}{l}\text { Mean : } 6 \\
\text { SD : } 1.7\end{array}$ \\
\hline $\begin{array}{l}\text { Yu et al., } \\
2012\end{array}$ & $\begin{array}{l}\text { Korea } \\
\text { Selatan }\end{array}$ & CP hemiplegic & $\begin{array}{l}\text { WeeFIM- } \\
\text { SC }\end{array}$ & CIMT & $\begin{array}{l}\text { Mean : } 21.7 \\
\text { SD : } 3.6\end{array}$ & $\mathrm{TT}$ & $\begin{array}{l}\text { Mean : } 20.6 \\
\text { SD : } 4.6\end{array}$ \\
\hline
\end{tabular}

\section{a. Forest plot}

Figure 4 shows that the results of the analysis show that giving CIMT to children with cerebral palsy can improve occupational performance abilities by 0.18 times than non-CIMT interventions. However, these results were not statistically significant $(\mathrm{SMD}=0.18 ; 95 \% \mathrm{CI}-0.17$ to $0.52 ; \mathrm{p}=$ 0.32).

\begin{tabular}{|c|c|c|c|c|c|c|c|c|c|c|c|}
\hline \multirow[b]{2}{*}{ Study or Subgroup } & \multicolumn{3}{|c|}{ Experimental } & \multicolumn{3}{|c|}{ Control } & \multicolumn{2}{|r|}{ Std. Mean Difference } & \multirow{2}{*}{\multicolumn{3}{|c|}{$\begin{array}{c}\text { Std. Mean Difference } \\
\text { IV, Random, } 95 \% \mathrm{Cl}\end{array}$}} \\
\hline & Mean & SD & Total & Mean & SD & Total & Weight & IV, Random, $95 \% \mathrm{Cl}$ & & & \\
\hline Aarts 2010 & 6.5 & 1 & 28 & 4.6 & 1.4 & 22 & $9.5 \%$ & $1.57[0.93,2.21]$ & & & \\
\hline Brandao 2010 & 74.46 & 9.88 & 8 & 69.15 & 6.31 & 8 & $6.4 \%$ & $0.61[-0.40,1.61]$ & & & \\
\hline Brandao 2012 & 5.54 & 1.7 & 8 & 6.58 & 1.19 & 8 & $6.3 \%$ & $-0.67[-1.69,0.35]$ & & & \\
\hline Chen 2014 & 42.61 & 6.24 & 23 & 39.73 & 3.66 & 22 & $10.0 \%$ & $0.55[-0.05,1.15]$ & & & \\
\hline Deppe 2013 & 62.2 & 8.1 & 24 & 63.2 & 8.3 & 18 & $9.8 \%$ & $-0.12[-0.73,0.49]$ & & & \\
\hline Fedrizzi 2013 & 2.4 & 0.95 & 39 & 2.38 & 0.83 & 33 & $11.3 \%$ & $0.02[-0.44,0.49]$ & & & \\
\hline Hwang 2020 & 17.92 & 12.47 & 12 & 16.25 & 9.27 & 12 & $8.0 \%$ & $0.15[-0.65,0.95]$ & & & \\
\hline Sakzewski 2011 & 6.3 & 1.9 & 29 & 6.3 & 1.5 & 29 & $10.8 \%$ & $0.00[-0.51,0.51]$ & & & \\
\hline Sakzewski 2015 & 5.7 & 1.5 & 27 & 6.5 & 1.6 & 20 & $10.1 \%$ & $-0.51[-1.10,0.08]$ & & & \\
\hline Wallen 2011 & 6.1 & 2.3 & 25 & 6 & 1.7 & 25 & $10.4 \%$ & $0.05[-0.51,0.60]$ & & & \\
\hline Yu 2012 & 21.7 & 3.6 & 10 & 20.6 & 4.6 & 10 & $7.4 \%$ & $0.26[-0.63,1.14]$ & & & \\
\hline Total $(95 \%$ Cl) & & & 233 & & & 207 & $100.0 \%$ & $0.18[-0.17,0.52]$ & & & \\
\hline $\begin{array}{l}\text { Heterogeneity: Tau }{ }^{2} \\
\text { Test for overall effec }\end{array}$ & $\begin{array}{l}0.22 ; \mathrm{Cl} \\
\mathrm{Z}=1.00\end{array}$ & $\begin{array}{l}h i^{2}=30 \\
(P=0 .\end{array}$ & $\begin{array}{l}06, d f= \\
32)\end{array}$ & $10(P=$ & $=0.000$ & 08); $\left.\right|^{2}=$ & $=67 \%$ & & -2 & non CIMT CIMT & 2 \\
\hline
\end{tabular}

Figure 4. Forest plot of CIMT's influence on performance occupation of children with cerebral palsy 


\section{b. Funnel plot}

In Figure 5, the funnel plot graph looks symmetrical, which shows that there is no

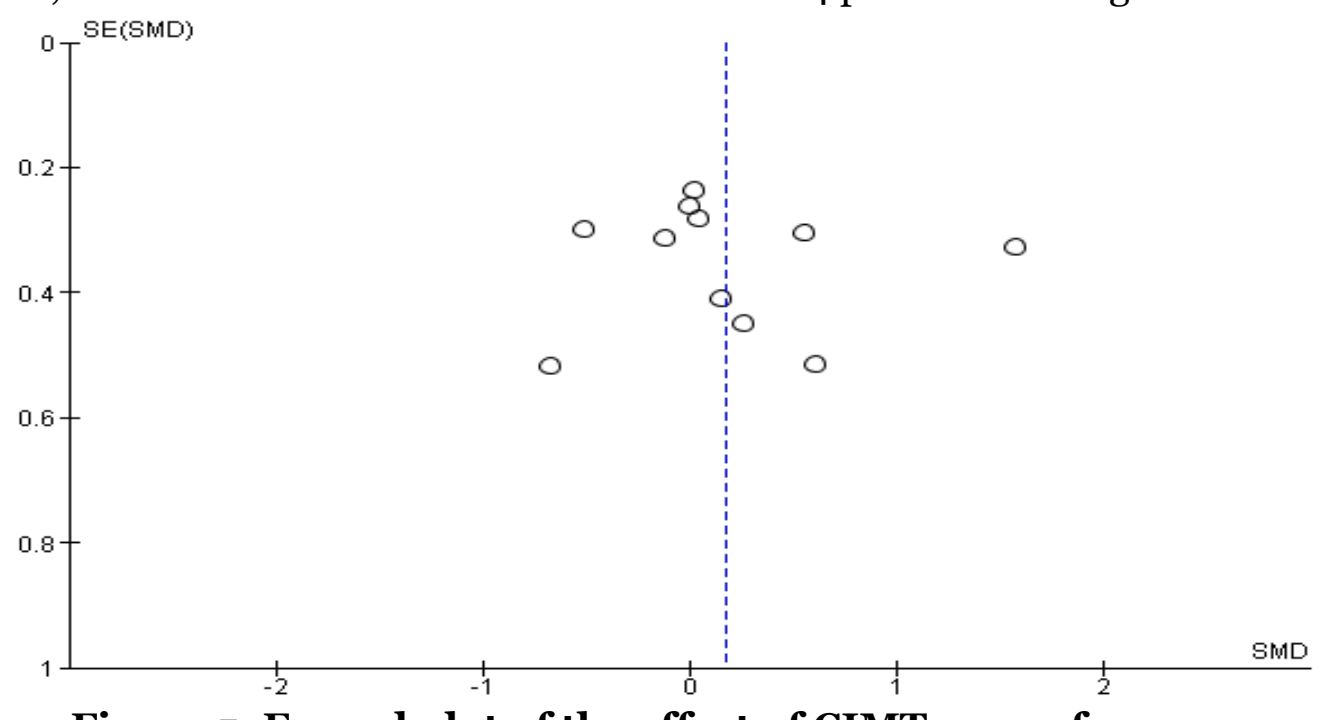

Figure 5. Funnel plot of the effect of CIMT on performance occupation of children with cerebral palsy

\section{DISCUSSION}

Cerebral palsy is a stable condition and a non-progressive disorder in the brain of a fetus or developing baby so that they are considered normal children with special needs (Levitt and Addison, 2019; Miller et al., 2020). In children with cerebral palsy, limited hand function is a common consequence of spasticity which complicates the child's fine motor movements such as grasping, writing, and cutting (Klevberg et al., 2017; Duruöz, 2019).

Some experts say that CIMT is a rehabilitation technique in the upper limb with a focus on improving the function of the affected limb (Miller et al., 2020). In this technique, repetitive motion exercises are given so that this can provide movement memory to the child. According to Arenz et al., (2017), these repetitive or repetitive movements can generate or create new neural pathways in the brain.

This study uses a systematic review and meta analysis study design with the aim of obtaining general conclusions as the basis for providing therapy / intervention publication bias because there are 6 points on the left side, 1 point parallel to the line and 4 points on the right. from various similar studies that have been carried out by previous researchers who tested the administration of CIMT in children with cerebral palsy in various countries.

Meta analysis is an epidemiological study that combines and combines statistically primary research data that discusses the same hypothesis so as to obtain quantitative summary results (Egger and Smith in Murti, 2018). The results of the research are presented in the form of a forest plot and a funnel plot.

Forest plots can show effect sizes and 95\% confidence intervals or display the results of a meta-analysis study (Makowski et al., 2019). The funnel plot shows the effect size and precision of the effect size and makes it possible to evaluate the possibility of publication bias in the form of a symmetrical triangle graphic (Makowski et al., 2019; Li et al., 2020).

\section{Hand function}

Giving CIMT to children with cerebral palsy was significantly able to increase hand function by 0.4 times than non-CIMT 
interventions. The results of this study are supported by the research of Aarts et al (2010) where giving CIMT-BiT to children with cerebral palsy can improve children's hand function 0.4 times higher than giving intervention or standard therapy. The provision of CIMT intervention also provides significant results on the home base model and school base model (Al-Oraibi and Eliasson, 2011; Gelkop et al., 2015).

Giving pure and combined CIMT provides benefits to weak sensory function even though it does not improve hand structure but can improve the ability to move the arms, hands and hand coordination of children with hemiplegi (SimonMartinez et al., 2020; Wallen et al., 2011).

Comparison of CIMT doses (3 or 6 hours) did not provide a significant difference in results (Deluca et al., 2012). Basically, CIMT as a reference frame for occupational therapy is used in children because each exercise given is a play component that encourages the use of upper limb functionalities. Hemiplegic children to facilitate bimanual or upper limb performance (Arenz et al., 2017).

\section{Occupational performance}

Giving CIMT to children with cerebral palsy can improve occupational performance ability by 0.18 times than non-CIMT intervention although it is not statistically significant. In the research of Sakzewski et al. (2015) also wrote that giving hybrid-CIMT can improve occupational performance abilities even though this is not statistically significant.

However, there are differences in the research of Brandao, Gordon and Mancini (2012) which states that the provision of CIMT intervention can improve the ability of children's occupational performance and is statistically significant $(\mathrm{p}<0.001)$ by using the COPM instrument.
The target of giving active CIMT intervention includes the ability in activity daily living such as bathing, defecating, dressing, eating, or cleaning and grooming; IADL such as raising animals, taking formal lessons, and playing (Arenz et al., 2017). When absorbing the ICF model in CIMT for children, the expected result of giving CIMT is to increase continuity in carrying out daily activities using the intervened domain or hand (Miller et al., 2020).

In longitudinal studies, CIMT showed results with a high functional level after approximately 3.5 years but this development was also influenced by the type of brain lesion (Eliasson and Holmefur, 2015). In addition, differences in age groups do not adequately affect the results of interventions on the aspect of occupational performance (Chen et al., 2015).

The limitation in this study is that there is still a language bias. It is hoped that further research can carry out research using a larger number of subjects and a higher period of time and follow-up. In addition, primary research on the effects of CIMT has never been carried out in Indonesia.

\section{AUTHOR CONTRIBUTION}

Mutiana Agustin Sholikah is the principal researcher who selects topics, explores and collects research data. Agus Kristiyanto and Hanung Prasetya analyzed data and reviewed research documents.

\section{CONFLICT OF INTEREST}

There is no conlict of interest in this study.

\section{FUNDING AND SPONSORSHIP}

This study is self-funded. 


\section{ACKNOWLEDGEMENT}

We are very grateful to the database providers PubMed, Science Direct, AJOT, Springer Link, and Google Scholar.

\section{LIST OF ABBREVIATIONS}

AHA $=$ The Assisting Hand Assessment

BiT = Bimanual Therapy

BOTMP = The Bruininks-Oseretsky Test of Motor Proficiency

CEBM = The Centre for Evidence-Based Medicine

CI = Confidence Interval

CIMT = Constraint Induced Movement Therapy

$\mathrm{COPM}=$ Canadian Occupational Performance Measure

$\mathrm{CP}=$ Cerebral Palsy

HABIT = Hand-Arm Bimanual Intensive Therapy

JTHF = Jebsen-Taylor Hand Function

JTHFT $=$ The Jebsen-Taylor Hand Function Test

mCIMT $=$ Modification Constraint Induced Movement Therapy

mCIT = Modification Constraint Induced Therapy

NDT $=$ Neuro Developmental Treatment

PEDI = Pediatric Evaluation of Disability Inventory

PICO = Population, Intervention, Control/Comparisons, Outcomes

PMAL = The Pediatric Motor Activity Log PMAL-AOU $=$ The Pediatric Motor Activity Log Amount of Use

PMAL-HW= The Pediatric Motor Activity Log- How Well

QUEST = Quality of Upper Extremity Skills Test

RCT = Randomized controlled trial

RevMan $=$ Review Manager

SMD = Strandart Mean Difference
VOAA-DDD=Video Observations Aarts and Aarts- Determine Developmental

WeeFIM= Wee-Funtional Independence Measure

\section{REFERENCE}

Aarts PB, Jongerius PH, Geerdink YA, Limbeek JV, Geurts AC (2010). Effectiveness of modified constraint-induced movement therapy in children with unilateral spastic cerebral palsy: A randomized controlled trial, Neurorehabil Neural Repair, 24(6): 509-518. doi: 10.1177/1545968309359767.

Al-Oraibi S, Eliasson AC (2011). Implementation of constraint-induced movement therapy for youngchildren with unilateral cerebral palsy in Jordan: A home-based model, Disabil Rehabil, 33(21-22): 2006-2012. doi: 10.3109/09638288.2011.555594.

AOTA (2014). The occupational therapy practice framework: Domain process 3rd Edition, Am J Occup Ther, 68: 69-75.

Arenz N, McCain K, Tjelta R (2017). There is moderate evidence that a low dose of CIMT (defined as 3 hours/day or less) is at least as effective as the traditional protocol involving 6 hours/day in improving upper extremity motor function in children with unilateral cerebral palsy. Available at: www.UWLAX.EDU/OT.

Brandao MdeB, Gordon AM, Mancini MC (2012). Functional impact of constraint therapy and bimanual training in children with cerebral palsy: A randomized controlled trial, Am J Occup Ther, 66(6): 672-681. doi: 10.5014/ajot.2012.004622.

Chen HC, Kang LJ, Chen CL, Lin KC, Chen FC, Wu KPH (2015). Younger children with cerebral palsy respond better 
than older ones to therapist-based constraint-induced therapy at home on functional outcomes and motor control, Phys Occup Ther Pediatr, 36(2): 1-14. doi: 10.3109/01942638.2015.1101042.

Christmas PM, Sackley C, Feltham MG, Cummins C (2018). A randomized controlled trial to compare two methods of constraint-induced movement therapy to improve functional ability in the affected upper limb in pre-school children with hemiplegic cerebral palsy: CATCH TRIAL, Clin Rehabil, 32(7): 909-918. doi: 10.1177/0269215518763512.

Deluca SC, Case-Smith J, Stevenson R, Ramey SL (2012). Constraint-induced movement therapy (CIMT) for young children with cerebral palsy: Effects of therapeutic dosage, J Pediatr Rehabil Med, 5(2): 133-142. doi: 10.3233/PRM-2012-0206.

Duruöz MT (2019). Hand Function A Practical Guide to Assessment. Second Edi, Springer Nature Switzerland AG. Second Edi. Edited by M. T. Duruöz. Switzerland: Springer Nature Switzerland. doi: 10.1007/978-3-03017000-4_7.

Eliasson AC, Holmefur M (2015). The influence of early modified constraintinduced movement therapy training on the longitudinal development of hand function in children with unilateral cerebral palsy, Dev Med Child Neurol, 57(1): 89-94. doi: 10.1111/dmcn.12589.

Gelkop N, Burshtein DG, Lahav A, Brezner A, Al-Oraibi S, Ferre CL, Gordon AM (2015). Efficacy of constraint-induced movement therapy and bimanual training in children with hemiplegic cerebral palsy in an educational setting, Phys Occup Ther Pediatr,
35(1): 24-39. doi: 10.3109/01942638.2014.925027.

Gulati S, Sondhi V (2018). Cerebral Palsy: An Overview, Indian J Pediatr, 85(11): 1006-1016. doi: 10.1007/s12098-0172475-1.

Ilieva E, Ilieva A (2020). What is the effect of constraint-induced movement therapy on children with unilateral cerebral palsy? A Cochrane Review summary with commentary, Dev Med Child Neurol, 62(11): 1236-1238. doi: 10.1111/dmen.14676.

Klevberg GL, Østensjø S, Elkjær S, Kjeken I, Jahnsen RB (2017). Hand Function in Young Children with Cerebral Palsy: Current Practice and Parent-Reported Benefits, Phys Occup Ther Pediatri, 37(2): 222-237. doi: 10.3109/01942638.2016.1158221.

Levitt S, Addison A (2019). Treatment of Cerebral Palsy and Motor Delay. sixth edit. Hoboken, NJ: Wiley-Blackwell. doi: 10.1136/jnnp.61.1.121-b.

Li G, Zeng J, Tian J, Levine MA, Thabane L (2020). Multiple uses of forest plots in presenting analysis results in health research, J Clin Epidemiol, 117: 89-98. doi:10.1016/j.jclinepi.2019.09.021.

Makowski D, Piraux F, Brun F (2019). From Experimental Network to Metaanalysis Methods and Applications with R for Agronomic and Environmental Sciences, France: Springer Nature B.V.

Miller F, Bachrach S, Lennon N, O'Neil ME (2020). Cerebral Palsy. 2th edn. Cham, Switzerland: Springer Nature Switzerland AG. doi: https://doi.org/10.1007/978-3-319-74558-9.

Murti B (2018). Prinsip dan Metode Riset Epidemiologi. edisi IV. Surakarta: Program Studi Ilmu Kesehatan Masyarakat, Program Pascasarjana, 
Sholikah et al./ the Effect of Constraint-Induced Movement Therapy on Hands Functional Ability

Universitas Sebelas Maret.

Sakzewski L, Miller L, Ziviani J, Abbott DF, Rose S, Macdonell RAL, Boyd RN (2015). Randomized comparison trial of density and context of upper limb intensive group versus individualized occupational therapy for children with unilateral cerebral palsy, Dev Med Child Neurol, 57(6): 539-547. doi: 10.1111/dmcn.12702.

Simon-Martinez C, Mailleux L, Hoskens J, Ortibus E, Jaspers E, Wenderoth N, Sgandurra G, et al. (2020) Randomized controlled trial combining constraint-induced movement therapy and action-observation training in unilateral cerebral palsy: clinical effects and influencing factors of treatment response, Ther Adv Neurol Disord, 13(1): 1-19. doi: 10.1177/1756286419898065.

Wallen M, Ziviani J, Naylor O, Evans R, Novak I, Herbert R (2011). Modified constraint-induced therapy for children with hemiplegic cerebral palsy: A randomized trial, Dev Med Child Neurol, 53(12): 1091-1099. doi: 10.1111/j.1469-8749.2011.04086.x. 